\title{
The Effect of Cervical Sympathectomy on Retinal Vessel Responses to Systemic Autonomic Stimulation
}

\author{
L. P. LANIGAN, ${ }^{*}$ R. BIRCHE, $\dagger$ C. V. CLARK,
}

\begin{abstract}
Summary
The retinal vessel calibre responses to systemic sympathetic stimulation, were studied in nine patients (eight male; mean age: 31.7 years; range: $19-58$ years) with unilateral disruption of their cervical sympathetic tract. All patients had ipsilateral decreased/absent facial sweating and a Horners syndrome, evidence of unilateral sympathetic denervation. Both eyes of each patient were studied and the results were analysed in two groups: the group of nine sympathectomised eyes and the control group of unaffected fellow eyes. During handgrip contraction there was a significant difference in the mean retinal arteriolar constriction (mean \pm SEM) between the group of sympathectomised eyes $(4.6 \pm 0.89 \%)$ and control eyes $(7.1 \pm 1.13 \%)$, $\mathbf{p}<\mathbf{0 . 0 1}$. Similarly, there was a significant difference in mean venule constriction during sustained handgrip contraction between the group of sympathectomised eyes $(1.5 \pm 0.67 \%)$ and control eyes $(4.9 \pm 0.98 \%), p<0.05$. There was no significant difference in the mean rise in diastolic blood pressure between the two groups: control eyes $+27.9 \pm 2.38 \mathrm{mmHg}$ and sympathectomised eyes $+27.8 \pm 2.25 \mathrm{mmHg}$. There was no correlation between the blood pressure and retinal vessel responses in either group.

These results suggest that the sympathetic nervous system plays an integral role in retinal blood flow regulation.
\end{abstract}

Until comparatively recently, there have been conflicting reports both supporting and denying the existence of autonomic innervation to the retinal vasculature. In 1963, Laties and coworkers, ${ }^{1}$ using a fluorescent histochemical method, reported that the nerve supply to the central retinal artery in the rabbit did not extend beyond the lamina cribrosa on entering the globe. Similar results were obtained from studies involving the retinal arterioles of both New and Old World monkeys and cats. ${ }^{2}$ By contrast, Seitz in 1953 had demonstrated vasodilatation of the rabbit pre-retinal vessels after bilateral superior cervical ganglionectomy, suggesting that sympathetic nerves were present in the pre-retinal vessels of the rabbit eye. ${ }^{3}$ In a study of the neuropathy of the retina and the optic nerve in diseased human eyes, Matsuyama demonstrated nerve fibres running parallel with retinal vessels using light microscopy; ${ }^{4}$ some of the nerve fibres followed the same direction as the retinal blood vessels prompting the hypothesis of centrifugal nerve fibres in the retina, but the origin of these nerve fibres was not discussed. Unmyelinated nerve fibres with vesicles have subsequently been demonstrated in the retinal vessel adventitia. ${ }^{5,6}$ Adrenergic nerve fibres have also been observed in the wall of human retinal vessels on the optic disc beyond

From: * Research Department of Ophthalmology, Royal College of Surgeons of England; †St Marys Hospital and $\ddagger$ Moorfields Eye Hospital, London.

Correspondence to: L. P. Lanigan, FRCSE, Moorfields Eye Hospital, City Road, London EC1V 2PD. 
the lamina cribrosa and for several millimetres proximally on the retinal surface surrounding the optic disc using transmission electron microscopic ${ }^{7}$ and fluorescent examination. ${ }^{8}$ Furukawa et al., ${ }^{9}$ using scanning electron microscopy and transmission electron microscopy, have detected unmyelinated nerve fibres with nerve endings filled with vesicles in retinal arterioles in man, rabbit and rat, supporting the concept of autonomic innervation to the retinal vasculature.

Recent reports have shown a consistent response of the retinal vessels to systemic autonomic nerve stimulation in normal subjects, with a mean constriction of retinal arterioles of $8.1 \%$ and retinal venules of $3.7 \%$, in a control group. ${ }^{10}$ By contrast, retinal vasoconstriction in response to sustained isometric muscle contraction was significantly impaired in patients with diabetes mellitus, ${ }^{11}$ the commonest cause of autonomic neuropathy in the United Kingdom. ${ }^{12}$ The aim of the present study was to assess the retinal vessel responses to systemic sympathetic nerve stimulation in patients with unilateral disruption of cervical sympathetic nerve innervation.

\section{Patients and Methods}

Nine patients with unilateral disruption of the cervical sympathetic tract were included in the study: eight were male, mean age 31.7 years, range $19-58$ years. Seven were victims of motorcycle accidents and had complete unilateral brachial plexus injuries (avulsion of cervical nerve roots, confirmed on surgical exploration several weeks after the accident). The remaining two patients had a surgical sympathectomy performed for relief of intractable pain (cervical neuritis and malignant schwannoma of the median nerve). Unilateral decreased or absent facial sweating on the affected side and unilateral Horner's syndrome (indicating sympathetic denervation) were present in all patients. The mean time interval from the date of their accidents to the date of this study was 2.2 years; with a range of one to four years.

All patients were studied following informed consent. The protocol ${ }^{10}$ was approved by the hospital ethical committee. Each patient was studied seated at the fundus camera with both pupils dilated with guttae tropicamide $1 \%$. All photographs were taken by the same observer using a wide angle Canon camera (CF 60-S) which was triggered during the diastolic phase of the pulse cycle. Each phase of photographs consisted of five photographs were taken in quick succession, at approximately one second intervals. A baseline phase of photographs was taken, followed by three baseline blood pressure measurements recorded from the non-exercising arm using an automatic sphygmomanometer (COPAL-UA 231). The patient was then instructed to perform three maximum handgrip contractions to establish his maximum voluntary contraction (MVC). A laboratory-built strain-gauge dynamometer with continuous chart recording was used with an adjustable audible alarm, set to alarm below $33 \%$ of each patient's MVC. The patient was then instructed to perform a sustained handgrip at $33 \%$ MVC for 2.5 minutes; three blood pressure measurements were recorded at $0.5,1.5$ and 2.5 minutes and three phases of fundus photographs were taken at 1 , 2 and 2.5 minutes during the handgrip contraction. On recovery one final phase of fundus photographs and one blood pressure reading were taken five minutes following release of handgrip contraction. All photographs were recorded on Ilford FP4 film with a red free filter and developed in PQ Universal $(1+19)$ for 12 minutes at $20^{\circ} \mathrm{C}$ with sensitometric control. ${ }^{13}$

As two patients had an above elbow amputation and another had the affected arm in a plaster cast (nerve transfer procedure done 4 weeks previously), the blood pressure readings were taken from the exercising arm, which was not always the dominant arm for obvious reasons; this convention was followed for all patients in order to standardise the procedure.

Both eyes of each patient were studied with a 15 minute interval between studies. The photographer randomly selected the order of the eyes to be studied for each patient. This permitted the sympathectomised eye to be compared with the control eye within each patient.

\section{Analysis}

All films were analysed using the Quantimet 


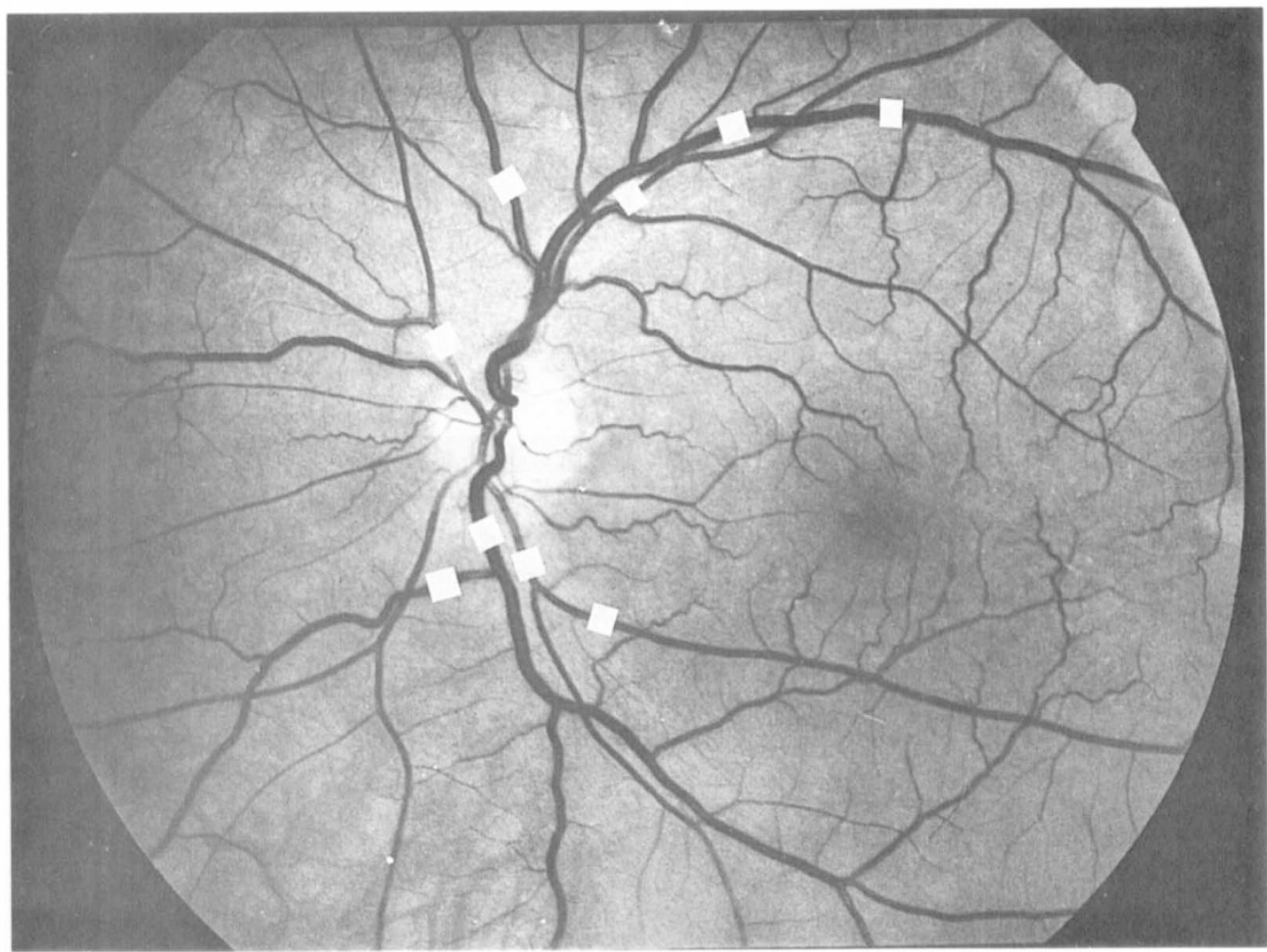

Fig. 1. Fundus photograph showing measurement sites.

800 Image analyser (Cambridge Instruments). ${ }^{13}$ Sixteen to 18 suitable vessel sites were selected from each fundus photograph for each eye, comprising approximately equal numbers of arterioles and venules. A sample fundus photograph illustrating typical sites of

ARTERIES

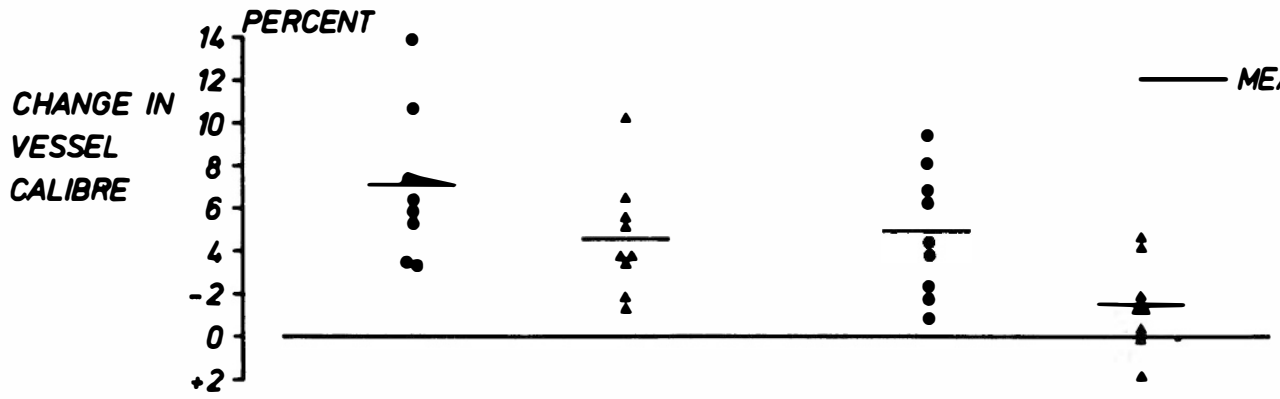

CONTROL SYMP. CONTROL SYMP.

Fig. 2. Individual mean vessel responses during handgrip contraction for control and sympathectomised eyes. measurement is shown in Figure 1. The following parameters were calculated for all calibre measurements: mean, standard error of mean (SEM) and one way analysis of variance within replications and between the phases for each site. Percentage change in 


\section{CONTROL SIDE}

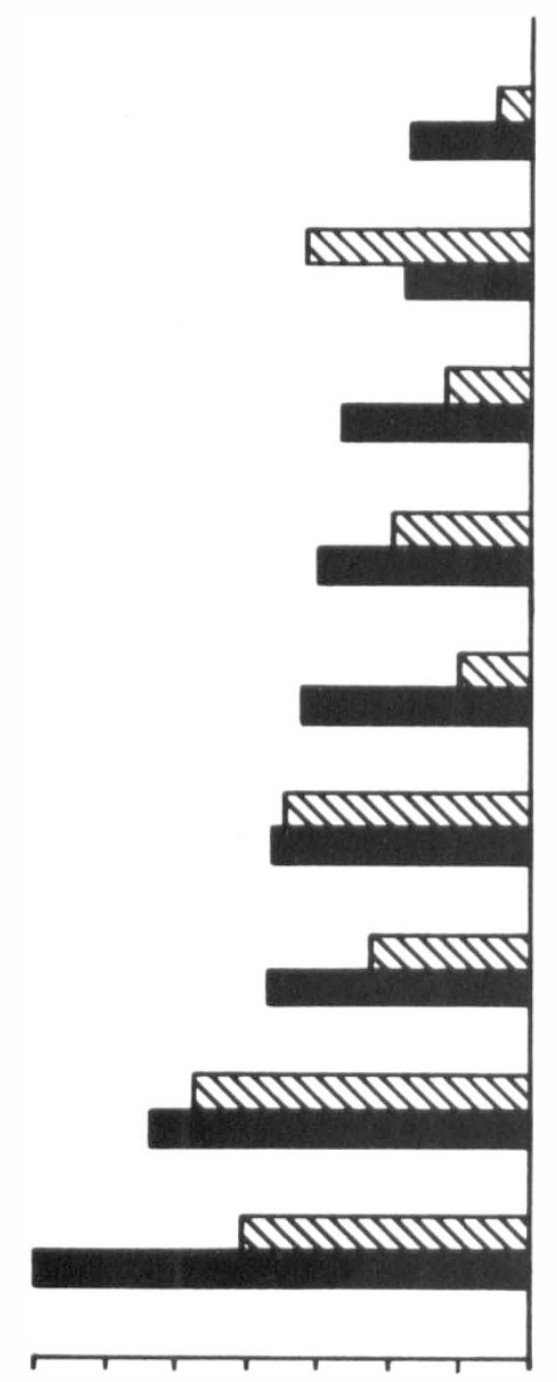

SYMPATHECTOMIZED SIDE

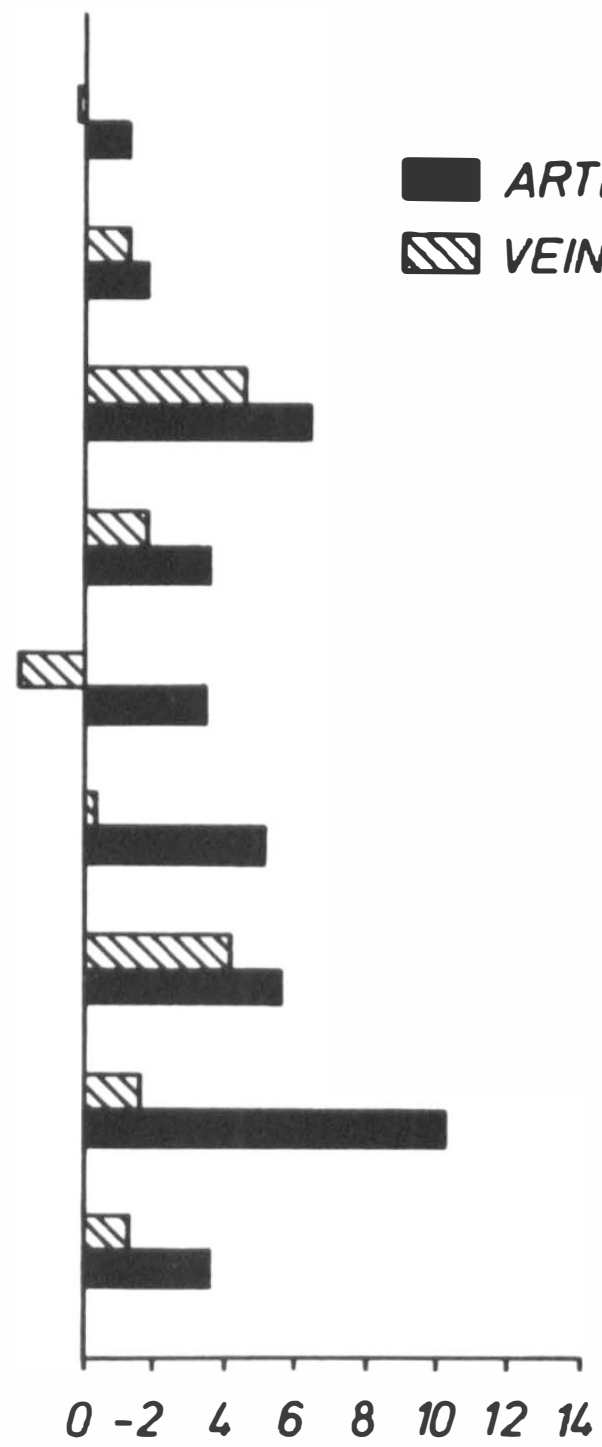

\section{MEAN \% CALIBRE CHANGE}

Fig. 3. Mean vessel responses during handgrip contraction in sympathectomised eyes compared with fellow control eyes for each subject.

mean retinal vessel calibre between phase 1 (control) and phase 4 (peak handgrip contraction) was calculated. Differences between individual mean vessel responses (unaffected and sympathectomised eyes) and between the two groups (control and sympathectomised groups) were assessed by the Wilcoxon signed rank test, with the level of statistical significance being taken at $\mathrm{p}<0.05$ for both arterioles and venules.

\section{Results}

The results of this study were analysed in two groups: the group of nine sympathectomised 


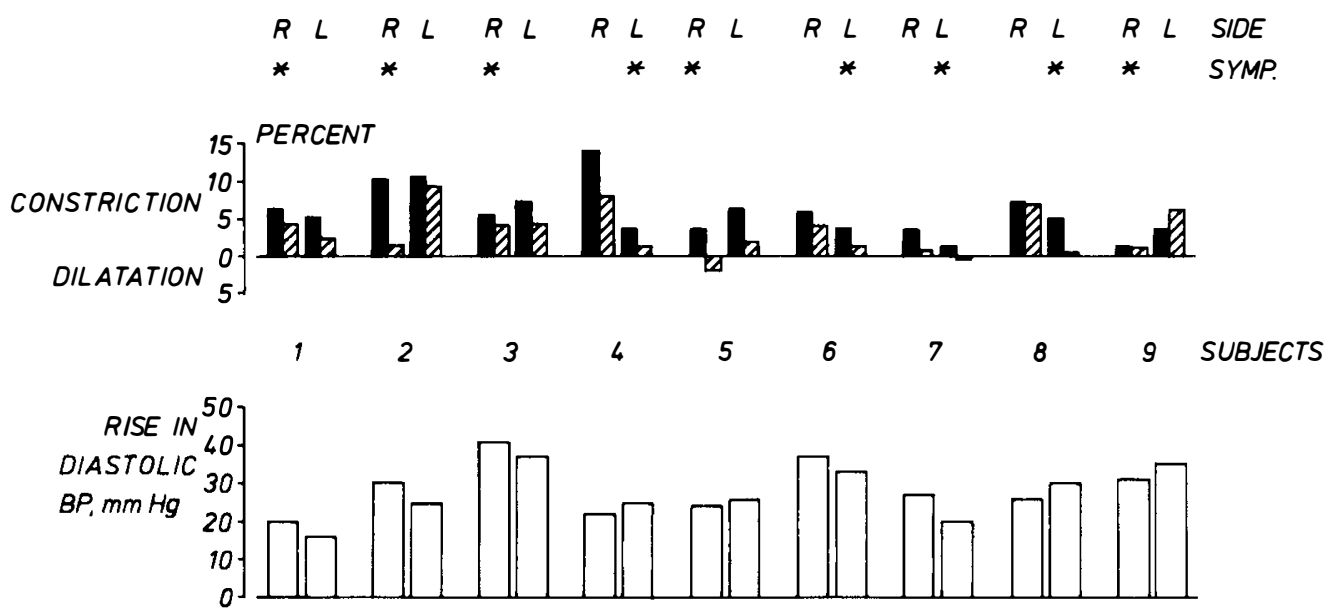

Fig. 4. Individual retinal vessel responses with corresponding blood pressure responses during handgrip contraction.

eyes and the control group of nine unaffected fellow eyes. A total of 166 retinal vessel sites were measured in both the control group ( 78 arteriolar and 78 venule sites) and in the sympathectomised group (82 arteriolar and 74 venular sites).

During the exercise period, there was a significant difference in the mean arteriolar retinal vessel constriction (mean \pm SEM) between the group of sympathectomised eyes $(4.6 \pm 0.89 \%)$ and control eyes $(7.1 \pm 1.13 \%)$, $\mathrm{p}<0.01$. Similarly there was a significant difference in mean venule constriction during sustained handgrip between the group of sympathectomised eyes $(1.5 \pm 0.67 \%)$ and control eyes $(4.9 \pm 0.98 \%), p<0.05$, (Fig. 2). The individual mean retinal vessel responses to sustained handgrip contraction for the sympathectomised and control fellow eyes, with the corresponding rise in diastolic blood pressure, are shown in Figs. 3 and 4; the range of retinal vessel responses to sustained handgrip contraction within the individual precluded statistically significant differences (between control and sympathectomised eyes), except for subject no. 4 (Fig. 4).

Figure 5 demonstrates the sequential change in mean retinal vessel calibre through phases 2 to 5 (handgrip contraction and recov- ery) compared to phase 1 (baseline), for sympathectomised and control eyes. While there was a gradually increasing degree of retinal vessel constriction with continued stimulation for both arteries and venules in both groups, there is nevertheless a definite reduction in the magnitude of the responses for the sympathectomised eyes.

There was no significant difference in the mean rise in diastolic blood pressure $(\mathrm{p}>0.05)$ between the two groups: control eyes, $+27.9 \pm 2.38 \mathrm{mmHg}$ and sympathectomised eyes, $+27.8 \pm 2.25 \mathrm{mmHg}$. There was no correlation between the blood pressure rise and the retinal arteriolar responses (controls, $\mathrm{r}=+0.30$ : sympathectomised eyes, $r=-0.22$ or the venule responses (control eyes, $r=0.0003$ : sympathectomised eyes, $\mathrm{r}=-0.33)$ in either group.

\section{Discussion}

Histological studies have confirmed the existence of autonomic receptors on retinal blood vessels, although the effects of these receptors are not known. Nerve endings on pre-retinal arterioles (fewer on venules), distributed from the optic disc to the periphery and containing axonal varicosities (diameter 0.5$1.5 \mu$ ) with vesicles, were demonstrated by 


\section{ARTERIES}

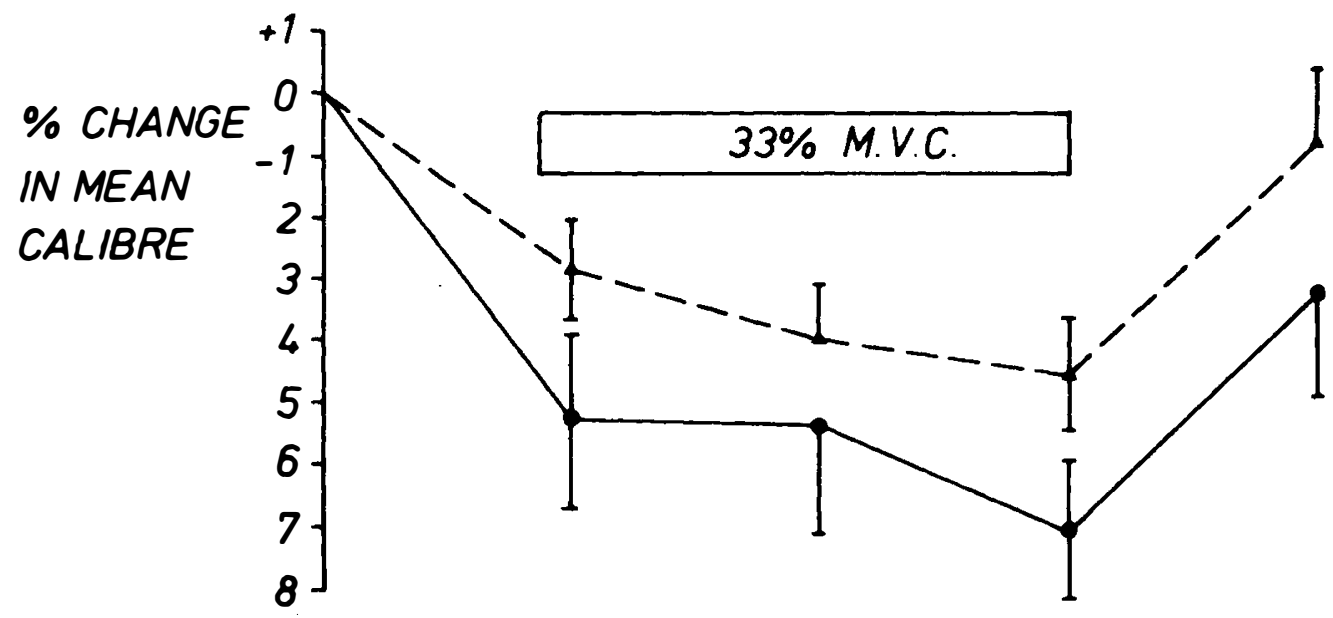

PHASE 1

2

3

4

5

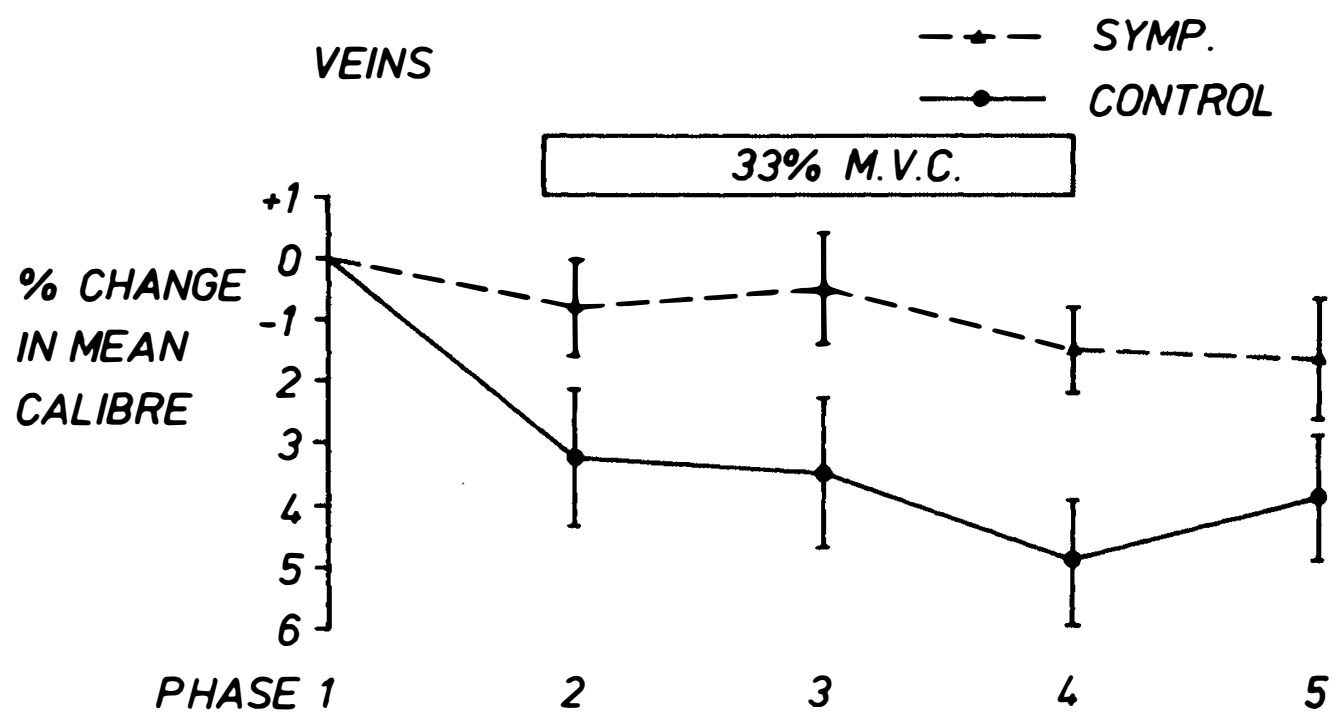

Fig. 5. Sequential change in retinal vessel constriction in sympathectomised and control eyes, during handgrip contraction.

Furukawa in 1987 in the rabbit eye. ${ }^{14}$ Using scanning electron microscopy, transmission electron microscopy and fluorescence histochemical examination, he showed an inverse relationship between both the number of nerve endings on arterioles and the diameter of the arteriole with distance from the optic nerve. The nerve endings were noted to disappear following superior cervical ganglionectomy, suggesting that sympathetic nerves 
originating in the superior cervical ganglion innervated pre-retinal vessels in the rabbit eye. Further evidence for autonomic innervation of the retinal vessels comes from a study by Forster, Ferrari-Dileo and Anderson, ${ }^{15}$ demonstrating the presence of autonomic binding sites in mammalian retinal vessels. The exact location of these sites on the vessels was not defined, however, as retinal vessel homogenates were used. High affinity alpha-1 and alpha-2 adrenergic binding sites were demonstrated both in the vessel homogenates and in the retinal tissue. This is not surprising as the retina is known to contain noradrenaline and is involved in its synthesis. ${ }^{16}$ In addition alpha adrenergic receptors can be stimulated by dopamine, which is present in high concentrations in the retina. Furthermore catecholaminergic amacrine cells are also present in the retina. ${ }^{1}$

The demonstration of alpha- 1 and alpha-2 high-affinity binding sites-presumably receptors-is also not surprising, as post-synaptic alpha receptors are present in peripheral and central aortic arteries as a mixed population of alpha- 1 and alpha- 2 receptors. Both subserve contractile responses, but the mechanism of each receptor is different. The alpha-2 receptor is negatively coupled to adenylate cyclase and is critically dependent on extracellular calcium ions to elicit its vasoconstrictor response ${ }^{17,18}$ whereas the alpha-1 type appears to be dependent on intracellular calcium ions and phosphatidylinositol turnover. ${ }^{19,20}$

The presence of adrenergic receptors in the retinal vessels may permit a contractile response to low concentrations of adrenergic agonists. As workers have recently suggested the presence of adrenergic innervation of the retinal vessels, there is therefore an inevitable potential for stimulation from the autonomic nervous system. Indeed the normal concentration of circulating catecholamines is in the nanomolar range, which is sufficient to stimulate alpha- 1 and alpha- 2 receptors and thus maintain smooth muscle tone efficiently.

The mechanism by which intraluminal catecholamines affect receptors on smooth muscle receptors is not known as retinal arterioles lack fenestrations and possess tight junctions in the endothelium. This suggests that recep- tor stimulation could only occur if there were a breach in the inner blood retinal barrier. An alternative explanation would be that the alpha receptors were located in the endothelium, rather than the muscle layer. Indeed evidence for alpha-2 receptors in the luminal layers of peripheral (mesenteric and renal) and central (aortic) arteries exists; ${ }^{21,22}$ these alpha-2 receptors promote a relaxing response or at least attenuate a pre-existing contractile one. A third explanation might be the presence of receptors in all layers of the retinal vessels, with differing effects depending upon the integrity of the pre-existent vascular anatomy.

The results of the present study demonstrate that cervical sympathectomised eyes show a significant decrease in group mean retinal vessel responses to systemic autonomic stimulation compared with fellow eyes with an intact sympathetic innervation. While these observations would tend to support recently reported work which has demonstrated adrenergic binding sites in mammalian pre-retinal vessels ${ }^{15}$ and autonomic nerve endings in retinal vessels,${ }^{14}$ interpretation of the results requires caution. Although mean retinal vessel responses to autonomic stimulation were significantly reduced in sympathectomised eyes, the arterial response was still present to a significant degree (a constriction of $4.6 \%$ ). This suggests that these retinal vessels may have an incomplete cervical sympathetic denervation-as indeed sympathetic nerves have a great propensity for regeneration-or that the sympathetic nerves are not the only factor responsible for autoregulatory responses. As retinal vessel responses to sustained handgrip may be an autoregulatory response to the rise in systemic blood pressure, both factors are important considerations.

Comparisons of retinal vessel responses in each sympathectomised eye with the corresponding fellow control eye for each individual patient demonstrated no significant differences in all but one patient. This may be due to the heterogeneous nature of the variables involved. For example although many sites were measured in each fundus, arteriolar and venule sites were selected randomly and cannot be assumed to be similar or identical to 
retinal sites in fellow eyes. Therefore although seven out of nine sympathectomised eyes showed a decrease in mean retinal arteriolar response to systemic autonomic stimulation (Fig. 3) when compared to their fellow unaffected eyes, the difference was not statistically significant, probably for the aforementioned reasons.

The results imply that the sympathetic nervous system exerts a significant effect-either directly or indirectly-on the smooth muscle tone of the retinal vasculature. Denervation may cause loss of tone and altered haemodynamics, and may therefore represent an important aetiological factor in the pathogenesis of diabetic retinopathy. Abnormal haemodynamics are well-recognised in the diabetic circulations; ${ }^{23-25}$ the effects of denervation in the retinal vasculature probably occurs as an integral feature of a generalised autonomic dysfunction. Indeed, Neubauer et al. showed considerable reduction in cardiovascular tissue concentrations of catecholamines in diabetic subjects, reflecting the extent of sympathetic denervation in these patients. ${ }^{26}$ Furthermore, a loss of significant retinal vessel responses to autonomic stimulation has recently been documented in diabetics with autonomic neuropathy, whilst retinal vessel responses to equivalent stimuli were normal in diabetic subjects with intact autonomic nerve function. ${ }^{11}$

The present study demonstrates a significant reduction in group mean retinal vessel responses to systemic autonomic nerve stimulation in sympathectomised eyes compared to control eyes, with the inevitable implication that impairment of vascular reflexes is related to disruption of cervical sympathetic innervation to these vessels. The results support the hypothesis that autonomic reflexes of the retinal vasculature may be a significant contributing factor in the effective autoregulation of retinal blood flow.

We are grateful to the patients for their cheerful participation in the study and to the Royal National Institute for the Blind for supporting the study. L. P. Lanigan held a Smith \& Nephew Fellowship in Ophthalmology.

\section{References}

${ }^{1}$ Laties AM and Jacobowitz D: A comparative study of the autonomic innervation of the eye in the monkey, cat and rabbit. Anat rec 1963, 156: 383-9.

${ }^{2}$ Laties AM: Central retinal artery innervation. Arch Ophthalmol 1967, 177: 405-9.

${ }^{3}$ Seitz R: Zur vegetativen innervation von pupille und netzhautgefaben. Ber Dtsch Ophthal Ges 1953, 58: 55-61.

${ }^{4}$ Matsuyama M: Observation on new-grown fibres in the optic nerve. Jpn J Ophthalmol 1961, 13: 105-16.

${ }^{5}$ Nomura T: Fine structure of the human retinal arteriole: a sphincter-like structure in the side arm branching and unmyelinated nerve fibres in the adventitia. Jpn J Ophthalmol 1972, 16: 158-66.

${ }^{6}$ Fukuda M: Presence of adrenergic innervation to the retinal vessels: a histochemical study. Jpn J Ophthalmol 1970, 14: 91-7.

${ }^{7}$ Ruskell GL: Dual innervation of the central artery of the retina in monkeys. In: The optic nerve, Kant JS ed, London, Kimpton 1972, 45-58.

${ }^{8}$ Ehinger B: Adrenergic nerves to the eye and to related structure in man and in the cynomolgus monkey (Macaca Irus). Invest Ophthalmol Vis Sci 1966, 5: 42-52.

${ }^{9}$ Furukawa H, Nomura T, Kurimoto S: Autonomic innervation of the retinal vessels in rabbit, human and rat eye. Nippon Ganka Gakkai Zashi 1984, 88(4): $668-75$.

${ }^{10}$ Lanigan LP, Clark CV, Hill DW: Retinal circulation responses to systemic autonomic nerve stimulation. Eye 1988, 2: 412-17.

${ }^{11}$ Lanigan LP, Clark CV, Allawi J, Hill DW, Keen H: Responses of the retinal circulation to systemic autonomic nerve stimulation in diabetes mellitus. Eye 1989, 3: 39-47.

${ }^{12}$ Watkins PJ and Edmonds ME: Clinical presentation of diabetic autonomic failure. In: Bannister R ed. Autonomic failure. Oxford, Oxford University Press 1983: 332.

${ }^{13}$ Hill DW and Crabtree A: Vascular calibres. Trans Ophthalmol Soc UK 1984, 104: 107.

${ }^{14}$ Furukawa $\mathrm{H}$ : Autonomic innervation of pre retinal blood vessels of the rabbit. Invest Ophthalmol Vis Sci 1987, 28: 1752-60.

${ }^{15}$ Forster BA, Ferrari-Dileo G, Anderson: Adrenergic alpha 1 and alpha 2 binding sites are present in bovine retinal blood vessels. Invest Ophthalmol Vis Sci 1987, 28: 1741-6.

${ }^{16}$ Cohen $\mathbf{J}$ and Hadjiconstantinou M: Identification of epinephrine and phenylethanolamine-n-methyl transmethyl transferase in rat retina. Fed Proc 1984, 433: 2725-28.

${ }^{17}$ Mathews W, Jim K, Hieble P, de Marinis R: Postsynaptic alpha adrenoreceptors on vascular smooth muscle. Fed Proc 1984, 43: 2923-28.

${ }^{18}$ Ruffolo RR: Interactions of agonists with peripheral alpha adrenergic receptors. Fed Proc 1984, 43: 2910-14.

${ }^{19}$ Fain JN and Garcia-Sainz JA: Role of the phosphatidylinositol turnover in alpha- 1 and of adenylate cyclase inhibition in alpha-2 effects of catecholamines. Life Sci 1983, 26: 1183-94.

${ }^{20}$ Zelexnikar RJ, Quist EE, Drewes LR: An alpha-1 adrenergic receptor mediated phosphatidylinositol effect in canine cerebral microvessels. Mol Pharmacol 1983, 24: 163-6.

${ }^{21}$ Cocks $M$ and Angus $\mathrm{J}$ : Endothelium dependent 
relaxation of coronary arteries by noredrenaline and serotonin. Nature 1983, 305: 627-30.

${ }^{22}$ Eglene C, Godfraind T, Miller RC: Advanced responsiveness of rat isolated aorta to clonidine after removal of the endothelial cells. Br J Pharmacol 1984, 81: 16-18.

${ }^{23}$ Rhie FH, Chriestlieb AR, Sandor T, Gleason RE, Rand LI, Shah ST, Soeldner JS: Retinal Vascular reactivity to norepinephrine and angiotensin 11 in normals and diabetics. Diabetes 1982, 31: $1056-62$.
${ }^{24}$ Kohner EM: The problems of retinal blood flow in diabetes. Diabetes 1976, 25 (Supplement 2): 839-44.

${ }^{25}$ Sinclair SH, Grunwald JE, Riva CE, Braunstein SN, Nichols CW, Schwartz SS: Retinal vascular autoregulation in diabetes mellitus. Ophthalmology, 1982, 89: 748-50.

${ }^{26}$ Neubauer B and Christensen NJ: The noradrenaline, adrenaline and dopamine content in the cardiovascular system in longterm diabetics. Diabetes 1976, 25: 6-10. 